\title{
Associations of Serum Phosphorus Concentrations with Insulin Resistance and Hyperandrogenemia in Normal Weight Women with Polycystic Ovary Syndrome
}

\section{Xiao-ke Wu ( $2417956638 @ q q . c o m)$}

Department of Obstetrics and Gynecology, First Affiliated Hospital, Heilongjiang University of Chinese Medicine

\section{Shi-ke Zhang}

Department of Rehabilitation Medicine, Southern University of Science and Technology Yantian Hospital Hui He

Heilongjiang University of Chinese Medicine

\section{Yu Wang}

Department of Obstetrics and Gynecology, First Affiliated Hospital, Heilongjiang University of Chinese Medicine

\section{Jian Li}

Department of Obstetrics and Gynecology, First Affiliated Hospital, Guizhou Medical University

\section{Jing-shu Gao}

Department of Obstetrics and Gynecology, First Affiliated Hospital, Heilongjiang University of Chinese Medicine

\section{Jing Cong}

Department of Obstetrics and Gynecology, First Affiliated Hospital, Heilongjiang University of Chinese Medicine Jia-xing Feng

Heilongjiang University of Chinese Medicine

\section{Meng-yi Zhu}

Heilongjiang University of Chinese Medicine

\section{Research Article}

Keywords: polycystic ovary syndrome, insulin resistance, hyperandrogenemia, endocrine, metabolism, phosphorus

Posted Date: March 2nd, 2022 
DOI: https://doi.org/10.21203/rs.3.rs-1384361/v1

License: (c) (1) This work is licensed under a Creative Commons Attribution 4.0 International License. Read Full License 


\section{Abstract}

Purpose: Serum phosphorus is reported to be associated with insulin resistance (IR) and testosterone in many populations. However, studies exploring the role of serum phosphorus in polycystic ovary syndrome (PCOS) patients are rare. Thus, we aimed to investigate the associations of serum phosphorus concentrations with IR and hyperandrogenemia (HA) in women with PCOS.

Methods: This was a secondary analysis of a multicenter and large-sample clinical trial including 1000 PCOS subjects diagnosed by the modified Rotterdam criteria. A total of 508 normal-weight PCOS patients with available serum phosphorus data were enrolled in the present study. Serum phosphorus, metabolic indices, and total testosterone (TT) concentration were measured. IR status was defined by a homeostasis model assessment of insulin resistance (HOMA-IR) $\geq 2.69$, and HA status was defined as a $\mathrm{TT}$ level $\geq 1.67 \mathrm{nmol} / \mathrm{L}$. Multivariable linear regression and logistic regression models were used to evaluate the associations of serum phosphorus with IR and HA.

Results: Multiple linear regression analysis showed that the serum phosphorus concentration was inversely correlated with fasting plasma glucose (FPG), fasting insulin (FIN), HOMA-IR, and TT ( $P$ trend < 0.05 for all) after adjusting for confounding factors. Logistic regression showed that the serum phosphorus concentration was negatively correlated with IR and HA status. The multivariable-adjusted odds ratios with $95 \%$ confidence intervals for IR and HA status for the lowest $v s$. the highest quartiles of serum phosphorus were $2.26(1.17-4.35, P$ trend $=0.009)$ and $2.58(1.48-4.51, P$ trend $=0.002)$, respectively.

Conclusion: Our results demonstrated that lower serum phosphorus concentrations were associated with higher risk of IR and worsened HA in normal-weight women with PCOS.

\section{Introduction}

Polycystic ovary syndrome (PCOS) is a highly prevalent reproductive and endocrine disorder among women of reproductive age, with a prevalence range of $6.1-19.9 \%$ depending on the diagnostic criteria used[1]. Oligo-amenorrhea, androgen excess, and polycystic ovarian morphology on ultrasound are the most typical features. In addition, PCOS is frequently associated with obesity, marked insulin resistance (IR), and long-term risk of glucose intolerance, type 2 diabetes mellitus (T2DM), metabolic syndrome (MetS), and cardiovascular events [2][3].

IR, which refers to an inadequate cellular response to insulin activity in target tissues, is a key characteristic of PCOS. Studies have shown that approximately $50-70 \%$ of PCOS patients show some degree of IR [4], and both overweight and lean PCOS patients suffer from defects in insulin signaling [5], and obesity is considered as a significant contributor to IR[6]. Furthermore, compensatory hyperinsulinemia $(\mathrm{HI})$ secondary to IR stimulates the production and secretion of androgens in ovarian theca cells and inhibits the generation of sex hormone-binding globulin (SHBG) in the liver, thus exacerbating hyperandrogenemia (HA) [7]. A recent study showed that IR/HI and HA play an 
indispensable role in the multisystemic pathophysiology of PCOS and contribute to the further development of metabolic abnormalities[7]. However, the exact molecular mechanism has not been completely elucidated. Therefore, greater insights into the etiology of IR and HA are of great significance for understanding the pathogenesis of PCOS.

Recently, altered levels of trace elements in PCOS patients have attracted great research interest [8]. Phosphorus, an essential element in the human body, is generally absorbed from the diet in the upper intestine and transported throughout the body to form bone, skeletal muscle, and soft tissues, and phosphorous levels are maintained through excretion and reabsorption in the kidney. Phosphorus is involved in a variety of cellular functions, including biochemical energy transfer, maintenance of genetic information, intracellular signaling, and membrane structural integrity[9], and phosphorus homeostasis may also be affected by changes in the levels of sex steroid hormones[10]. Therefore, unbalanced phosphorus homeostasis may lead to endocrine and metabolic dysfunction. Indeed, previous studies have reported that serum phosphorus levels are inversely associated with body mass index (BMI) [11], $\operatorname{IR}[12], T 2 D M[13]$, and MetS[14][15] in the general population. A negative correlation between serum phosphorus and testosterone $(T)$ level was also found in older men[16]. In addition, dietary phosphate intake is inversely associated with T deficiency in humans [17] and with IR in mice[18]. However, to the best of our knowledge, only one previous study reported a negative correlation relationship between serum phosphorus and IR in women with PCOS, and most of these patients were obese[19]. More studies are thus needed to explore the role of phosphorus in PCOS.

We performed this cross-sectional analysis to investigate the associations between serum phosphorus concentrations and metabolic parameters and T concentration in women with PCOS in order to provide further insight into the pathogenesis and management of PCOS. As mentioned above, serum phosphorus is inversely associated with $\mathrm{BMI}$, and obesity per se is also a well-recognized cause of IR and HA; therefore, to avoid the effect of obesity, only nonobese patients were included in the present study.

\section{Materials And Methods}

\section{Study Participants}

Data in the present study were derived from the Polycystic Ovary Syndrome Acupuncture and Clomiphene Trial (PCOSAct), a multicenter and randomized controlled trial conducted from 2012 to 2015 at 21 sites (27 hospitals) in mainland China, and a total of 1000 PCOS patients aged between 20 and 40 years old were enrolled to participate in the trial. All participants signed an informed consent for inclusion before participating. The study protocol was approved by the Ethics Committee in each site, and all methods were performed in accordance with the relevant guidelines and regulations. The trial was registered at Clinical Trials.gov (Number: NCT01573858) and chictr.org.cn (Number: ChiCTR-TRC-12002081). The details of the study design and the main results have been previously published elsewhere[20][21]. The present study was a secondary analysis of the PCOSAct, and out of the 1000 participants, 529 PCOS patients with normal weight were included in the present analysis. 
The diagnostic criteria for PCOS were established based on the modified Rotterdam criteria, including oligomenorrhea (defined as an intermenstrual interval $>35$ days or $<8$ menstrual bleedings in the past year) or amenorrhea (defined as an intermenstrual interval $>90$ days) together with biochemical/clinical hyperandrogenism (hirsutism defined by a modified Ferriman-Gallwey Score $\geq 5$ in the Chinese population) and/or polycystic ovaries (defined as $\geq 12$ antral follicles 2-9 $\mathrm{mm}$ in diameter or an ovarian volume $\geq 10 \mathrm{~cm}^{3}$ as determined by vaginal ultrasound). Exclusion criteria included other endocrine diseases, use of hormones or other medication in the past 3 months, pregnancy, postabortion or postpartum within the past 6 weeks, breastfeeding within the past 6 months, and not willing to sign the written consent.

\section{Anthropometric Measurements}

All participants underwent a complete physical examination at the baseline visit, including measurements of height, weight, waist circumference (WC), systolic blood pressure (SBP), and diastolic blood pressure (DBP). BMI was calculated as weight $(\mathrm{kg}) /$ height $\left(\mathrm{m}^{2}\right)$, and normal weight in the Chinese population is defined as a $\mathrm{BMI}<24 \mathrm{~kg} / \mathrm{m}^{2}$ [22].

\section{Biochemical Assays}

All blood samples were obtained on the morning of day 3 of the menstrual cycle after an overnight fast, stored at $-80^{\circ} \mathrm{C}$, and finally transported to the central laboratory at Heilongjiang University of Chinese Medicine to perform the analyses. Endocrine features included follicle stimulating hormone (FSH), luteinizing hormone (LH), estradiol (E2), progesterone (P), total testosterone (TT) and SHBG. The free androgen index (FAl) was calculated by the equation: $\mathrm{FAl}=\mathrm{TT}[\mathrm{nmol} / \mathrm{L}] / \mathrm{SHBG}[\mathrm{nmol} / \mathrm{L}] \times 100$. HA status was defined as TT $\geq 1.67 \mathrm{mmol} / \mathrm{L}$ according to the cutoff value in our laboratory. Metabolic parameters included fasting plasma glucose (FPG), fasting insulin (FIN), and the homeostasis model assessment of insulin resistance (HOMA-IR), which was calculated by the equation: $\mathrm{HOMA}-\mathrm{IR}=\mathrm{FIN}[\mathrm{mIU} / \mathrm{L}] \times \mathrm{FPG}$ $[\mathrm{mmol} / \mathrm{L}] / 22.5$. IR status was defined as HOMA-IR $\geq 2$.69[23]. Serum phosphorus levels were also measured.

FSH, LH, E2, P, TT, and FIN were analyzed by electro-chemiluminescence immune assay (ECLIA) (Roche Diagnostic, Basel, Switzerland), SHBG was analyzed by immunolite (Siemens Diagnostic, Munich, Germany), and FPG was analyzed by hexokinase assay (Maker, Chengdu, China). Serum phosphorus levels were measured by the direct molybdate method on an automatic biochemistry analyzer 7600 (Hitachi, Japan).

\section{Statistical analyses}

All data were analyzed by SPSS software (IBM SPSS, Inc., Chicago, IL, USA version 26.0), and continuous variables are presented as the mean \pm standard deviation. The serum phosphorus concentrations were categorized into four quartiles, and variables among the four quartiles were compared by tests for linear trends. Linear regression analysis was performed to assess the correlations between the variables and the serum phosphate concentration, and fully adjusted multiple linear regression was used to calculate 
the adjusted mean (95\% confidence interval $(\mathrm{Cl})$ ) for the associations between serum phosphorus (the independent variable) and the metabolic and androgen indices (the dependent variables). Because IR and $\mathrm{HA}$ are closely related, they are seen as confounders for each other. Regarding the metabolic parameters, we calculated the adjusted mean $(95 \% \mathrm{Cl})$ for FPG, FIN, and HOMA-IR across the quartiles of serum phosphorus concentrations. In model 1, we adjusted for age. In model 2, we additionally adjusted for BMI, WC, SBP, and DBP. In model 3, we further adjusted for FSH, LH, E2, TT and SHBG. Regarding androgen indices, we calculated the adjusted mean $(95 \% \mathrm{Cl})$ for TT and FAl across the quartiles of serum phosphorus levels. In model 1, we adjusted for age. In model 2, we additionally adjusted for BMI, WC, SBP, and DBP. In model 3, we further adjusted for FSH, E2, FPG, and FIN. Trends tests for associations of serum phosphorus levels with metabolic and androgen parameters were determined by reassigning the median value of each quartile to the corresponding category and modeling them as continuous variables. Considering the effect of potential confounders, multivariable logistic regression analysis was also used to calculate the adjusted odds ratios (ORs) with $95 \%$ Cls for IR and HA status associated with serum phosphorus concentrations. P-values $<0.05$ were considered statistically significant.

\section{Results}

A total of 529 normal-weight PCOS patients were enrolled, of which 21 participants with missing data on serum phosphorous were excluded, leaving 508 participants for analysis. The range of serum phosphorus concentrations in our subjects was $0.56-3.07 \mathrm{mmol} / \mathrm{L}$. The serum phosphorus level was classified into four quartiles (Q1: $<0.98, N=127 ; \mathrm{Q} 2$ : $0.98-1.12, \mathrm{~N}=143 ; \mathrm{Q} 3: 1.13-1.24, \mathrm{~N}=113$; and Q4: $>1.24, \mathrm{~N}=125)$, respectively.

The anthropometric and biochemical characteristics of the study participants across the quartiles of serum phosphate levels are shown in Table 1. DBP, FSH, TT, SHBG, FPG, FIN, and HOMA-IR decreased from the first to the fourth quartiles of serum phosphate levels. 
Table 1

Anthropometric and biochemical characteristics of study participants by quartiles of serum phosphorus

\begin{tabular}{|c|c|c|c|c|c|}
\hline \multirow[t]{2}{*}{ Variables } & \multicolumn{4}{|c|}{ Quartiles of Serum Phosphorus (mmol/L) } & \multirow[t]{2}{*}{$P_{\text {trend }}$} \\
\hline & Q1 $(<0.98)$ & $\begin{array}{l}\text { Q2 (0.98 - } \\
1.12)\end{array}$ & $\begin{array}{l}\text { Q3 (1.13 - } \\
1.24)\end{array}$ & $\mathrm{Q} 4(>1.24)$ & \\
\hline $\mathrm{N}$ & 127 & 143 & 113 & 125 & \\
\hline Age (years) & $27.60 \pm 2.94$ & $27.87 \pm 3.14$ & $27.35 \pm 3.18$ & $27.90 \pm 3.41$ & 0.754 \\
\hline $\mathrm{BMI}\left(\mathrm{kg} / \mathrm{m}^{2}\right)$ & $21.10 \pm 1.91$ & $20.91 \pm 1.88$ & $21.00 \pm 1.89$ & $21.15 \pm 1.97$ & 0.742 \\
\hline WC $(\mathrm{cm})$ & $78.79 \pm 7.79$ & $77.46 \pm 6.80$ & $78.59 \pm 8.59$ & $78.62 \pm 7.81$ & 0.822 \\
\hline $\mathrm{SBP}(\mathrm{mmHg})$ & $111.62 \pm 10.30$ & $109.78 \pm 10.04$ & $109.49 \pm 9.55$ & $110.34 \pm 8.75$ & 0.298 \\
\hline $\mathrm{DBP}(\mathrm{mmHg})$ & $74.88 \pm 8.06$ & $73.31 \pm 7.84$ & $72.74 \pm 7.63$ & $72.63 \pm 7.78$ & 0.021 \\
\hline $\mathrm{FSH}(\mathrm{mlU} / \mathrm{mL})$ & $6.61 \pm 1.99$ & $6.14 \pm 1.48$ & $6.12 \pm 1.67$ & $5.90 \pm 1.46$ & 0.001 \\
\hline $\mathrm{LH}(\mathrm{mlU} / \mathrm{mL})$ & $12.23 \pm 6.21$ & $12.34 \pm 6.54$ & $11.94 \pm 6.44$ & $11.29 \pm 6.55$ & 0.209 \\
\hline LH/FSH & $1.92 \pm 0.93$ & $2.09 \pm 1.23$ & $2.01 \pm 1.17$ & $1.95 \pm 1.02$ & 0.993 \\
\hline E2 (pmol/L) & $\begin{array}{l}288.32 \pm \\
298.75\end{array}$ & $\begin{array}{l}330.76 \pm \\
393.73\end{array}$ & $\begin{array}{l}280.33 \pm \\
311.64\end{array}$ & $\begin{array}{l}256.07 \pm \\
275.61\end{array}$ & 0.253 \\
\hline $\mathrm{P}(\mathrm{ng} / \mathrm{mL})$ & $2.64 \pm 3.19$ & $3.65 \pm 9.13$ & $3.25 \pm 7.69$ & $2.21 \pm 3.21$ & 0.489 \\
\hline TT (nmol/L) & $1.72 \pm 0.72$ & $1.66 \pm 0.62$ & $1.63 \pm 0.62$ & $1.52 \pm 0.55$ & 0.014 \\
\hline $\begin{array}{l}\text { SHBG } \\
(\mathrm{nmol} / \mathrm{L})\end{array}$ & $57.20 \pm 33.95$ & $52.47 \pm 31.55$ & $48.59 \pm 27.38$ & $49.60 \pm 29.86$ & 0.035 \\
\hline FAI & $4.16 \pm 3.07$ & $4.44 \pm 3.34$ & $4.84 \pm 4.25$ & $4.34 \pm 3.46$ & 0.530 \\
\hline FPG (mmol/L) & $5.17 \pm 0.86$ & $4.93 \pm 0.83$ & $4.86 \pm 0.71$ & $4.67 \pm 0.90$ & $\begin{array}{l}< \\
0.001\end{array}$ \\
\hline $\mathrm{FIN}(\mathrm{pmol} / \mathrm{L})$ & $86.28 \pm 86.14$ & $62.27 \pm 52.01$ & $69.65 \pm 83.49$ & $58.95 \pm 41.54$ & 0.007 \\
\hline HOMA-IR & $2.96 \pm 3.44$ & $2.05 \pm 2.05$ & $2.21 \pm 3.20$ & $1.84 \pm 1.48$ & 0.003 \\
\hline $\begin{array}{l}\text { BMI: body mas } \\
\text { pressure; FSH: } \\
\text { TT: total testos } \\
\text { plasma glucos }\end{array}$ & $\begin{array}{l}\text { רdex; WC: wais } \\
\text { icle stimulatin } \\
\text { one; SHBG: sex } \\
\text { IN: fasting ins }\end{array}$ & $\begin{array}{l}\text { umference; SE } \\
\text { mone; LH: lute } \\
\text { none binding } \\
\text { HOMA-IR: hom }\end{array}$ & $\begin{array}{l}\text { stolic blood } \\
\text { ig hormone; } \\
\text { lin; FAl: free } \\
\text { asis model a }\end{array}$ & $\begin{array}{l}\text { re; DBP: dias } \\
\text { radiol; P: prc } \\
\text { terone index, } \\
\text { nent of insul }\end{array}$ & $\begin{array}{l}\text { lood } \\
\text { rone; } \\
\text { fasting } \\
\text { stance }\end{array}$ \\
\hline
\end{tabular}

Linear regression analysis showed that serum phosphorus levels were significantly and negatively correlated with FSH $(\beta=-0.094, P=0.035)$, E2 $(\beta=-0.092, P=0.040)$, TT $(\beta=-0.092, P=0.039)$, FPG $(\beta$ $=-0.278, P<0.001), \mathrm{FIN}(\beta=-0.155, P=0.001)$, and HOMA-IR $(\beta=-0.173, P<0.001)$ and marginally negatively correlated with SHBG $(\beta=-0.088, P=0.051)$ (Supplementary Table S1). 
Multiple linear regression analysis showed that serum phosphorus concentrations were significantly and inversely associated with FPG, FIN, and HOMA-IR among all the models (Table 2). The adjusted means $(95 \% \mathrm{Cl})$ of FPG $(\mathrm{mmol} / \mathrm{L})$ in model 2 , from the lowest to the highest quartiles of serum phosphorus, were $5.16(5.01-5.30) \mathrm{mmol} / \mathrm{L}, 4.94(4.80-5.08) \mathrm{mmol} / \mathrm{L}, 4.87$ (4.71-5.02) $\mathrm{mmol} / \mathrm{L}$, and 4.67 (4.52-4.82) $\mathrm{mmol} / \mathrm{L}(P$ trend $<0.001)$, respectively. The corresponding values of FIN (pmol/L) were 86.16 (74.4697.85) pmol/L, 63.70 (52.67-74.74) pmol/L, 69.61 (57.16-82.05) pmol/L, and 57.48 (45.67-69.29) $\mathrm{pmol} / \mathrm{L}(P$ trend $=0.002)$, respectively. The corresponding values of HOMA-IR were $2.95(2.49-3.41), 2.11$ (1.68-2.54), $2.21(1.72-2.70)$, and $1.78(1.32-2.24)(P$ trend $=0.001)$, respectively. The associations were still significant in model 3 after further adjusting for FSH, LH, E2, TT and SHBG. 
Table 2

Adjusted means ( $95 \% \mathrm{Cl}$ ) of PFG, FIN, and HOMA-IR across quartiles of serum phosphorus

\begin{tabular}{|c|c|c|c|c|c|}
\hline \multirow{2}{*}{ Variables } & \multicolumn{4}{|c|}{ Quartiles of Serum Phosphorus (mmol/L) } & \multirow{2}{*}{$\begin{array}{l}P \\
\text { trend }\end{array}$} \\
\hline & Q1 $(<0.98)$ & Q2 (0.98 - 1.12) & Q3 $(1.13-1.24)$ & $\mathrm{Q} 4(>1.24)$ & \\
\hline Median & 0.87 & 1.07 & 1.17 & 1.36 & \\
\hline \multicolumn{6}{|l|}{ FPG } \\
\hline Model 1 & $\begin{array}{l}5.17(5.02- \\
5.32)\end{array}$ & $\begin{array}{l}4.93(4.79- \\
5.07)\end{array}$ & $\begin{array}{l}4.86(4.71- \\
5.02)\end{array}$ & $\begin{array}{l}4.67(4.53- \\
4.82)\end{array}$ & $\begin{array}{l}< \\
0.001\end{array}$ \\
\hline Model 2 & $\begin{array}{l}5.16(5.01- \\
5.30)\end{array}$ & $\begin{array}{l}4.94(4.80- \\
5.08)\end{array}$ & $\begin{array}{l}4.87(4.71- \\
5.02)\end{array}$ & $\begin{array}{l}4.67(4.52- \\
4.82)\end{array}$ & $\begin{array}{l}<.001 \\
0.001\end{array}$ \\
\hline Model 3 & $\begin{array}{l}5.13(4.98- \\
5.28)\end{array}$ & $\begin{array}{l}4.91(4.77- \\
5.05)\end{array}$ & $\begin{array}{l}4.86(4.71- \\
5.01)\end{array}$ & $\begin{array}{l}4.66(4.51- \\
4.81)\end{array}$ & $\begin{array}{l}<.001 \\
0.00\end{array}$ \\
\hline \multicolumn{6}{|l|}{ FIN } \\
\hline Model 1 & $\begin{array}{l}86.37(74.50- \\
98.24)\end{array}$ & $\begin{array}{l}62.08(50.86- \\
73.30)\end{array}$ & $\begin{array}{l}70.02(57.35- \\
82.68)\end{array}$ & $\begin{array}{l}58.74(46.73- \\
70.76)\end{array}$ & 0.003 \\
\hline Model 2 & $\begin{array}{l}86.16(74.46- \\
97.85)\end{array}$ & $\begin{array}{l}63.70(52.67- \\
74.74)\end{array}$ & $\begin{array}{l}69.61(57.16- \\
82.05)\end{array}$ & $\begin{array}{l}57.48(45.67- \\
69.29)\end{array}$ & 0.002 \\
\hline Model 3 & $\begin{array}{l}88.32(78.21- \\
98.44)\end{array}$ & $\begin{array}{l}61.78(52.39- \\
71.17)\end{array}$ & $\begin{array}{l}60.69(50.09- \\
71.30)\end{array}$ & $\begin{array}{l}57.72(47.62- \\
67.82)\end{array}$ & 0.001 \\
\hline \multicolumn{6}{|l|}{ HOMA-IR } \\
\hline Model 1 & $\begin{array}{l}2.96(2.50- \\
3.43)\end{array}$ & $\begin{array}{l}2.04(1.60- \\
2.48)\end{array}$ & $\begin{array}{l}2.22(1.73- \\
2.72)\end{array}$ & $\begin{array}{l}1.83(1.36- \\
2.30)\end{array}$ & 0.001 \\
\hline Model 2 & $\begin{array}{l}2.95(2.49- \\
3.41)\end{array}$ & $\begin{array}{l}2.11(1.68- \\
2.54)\end{array}$ & $\begin{array}{l}2.21(1.72- \\
2.70)\end{array}$ & $\begin{array}{l}1.78(1.32- \\
2.24)\end{array}$ & 0.001 \\
\hline Model 3 & $\begin{array}{l}3.03(2.63- \\
3.42)\end{array}$ & $\begin{array}{l}2.00(1.64- \\
2.37)\end{array}$ & $\begin{array}{l}1.93(1.52- \\
2.35)\end{array}$ & $\begin{array}{l}1.79(1.40- \\
2.19)\end{array}$ & 0.001 \\
\hline
\end{tabular}

Cl: confidence interval; FPG: fasting plasma glucose; FIN: fasting insulin; HOMA-IR: homeostasis model assessment of insulin resistance.

Model 1 was adjusted by age.

Model 2 was additionally adjusted by body mass index, waist circumference, systolic blood pressure and diastolic blood pressure.

Model 3 was additionally adjusted by follicle stimulating hormone, luteinizing hormone, estradiol, total testosterone and sex hormone binding globulin.

Test for trend was based on variable containing median value for each quartile.

Serum phosphorus concentrations were also significantly and inversely associated with TT among all models (Table 3). The adjusted means ( $95 \%$ Cls) of TT in model 2 across quartiles of serum phosphorus 
were $1.71(1.60-1.82) \mathrm{nmol} / \mathrm{L}, 1.65(1.55-1.76) \mathrm{nmol} / \mathrm{L}, 1.64(1.52-1.75) \mathrm{nmol} / \mathrm{L}$, and $1.52(1.41-1.64)$ $\mathrm{nmol} / \mathrm{L}(P$ trend $=0.011)$, respectively. Similar associations were observed in model 3 after further adjustment for FSH, E2, FPG, and FIN $(P$ trend $=0.015)$. However, there was no association between serum phosphate concentration and FAl in any of the models $(P>0.05$ for all).

Table 3

Adjusted means $(95 \% \mathrm{Cl})$ of $\mathrm{TT}$, and $\mathrm{FAl}$ across quartiles of serum phosphorus

\begin{tabular}{|c|c|c|c|c|c|}
\hline \multirow[t]{2}{*}{ Variables } & \multicolumn{4}{|c|}{ Quartiles of Serum Phosphorus (mmol/L) } & \multirow[t]{2}{*}{$P_{\text {trend }}$} \\
\hline & Q1 $(<0.98)$ & Q2 (0.98 - 1.12) & Q3 (1.13 - 1.24) & $\mathrm{Q} 4(>1.24)$ & \\
\hline Median & 0.87 & 1.07 & 1.17 & 1.36 & \\
\hline \multicolumn{6}{|l|}{ TT } \\
\hline Model 1 & $\begin{array}{l}1.72(1.61- \\
1.83)\end{array}$ & $\begin{array}{l}1.66(1.55- \\
1.76)\end{array}$ & $\begin{array}{l}1.63(1.51- \\
1.74)\end{array}$ & $\begin{array}{l}1.52(1.41- \\
1.64)\end{array}$ & 0.007 \\
\hline Model 2 & $\begin{array}{l}1.71(1.60- \\
1.82)\end{array}$ & $\begin{array}{l}1.65(1.55- \\
1.76)\end{array}$ & $\begin{array}{l}1.64(1.52- \\
1.75)\end{array}$ & $\begin{array}{l}1.52(1.41- \\
1.64)\end{array}$ & 0.011 \\
\hline Model 3 & $\begin{array}{l}1.73(1.62- \\
1.84)\end{array}$ & $\begin{array}{l}1.64(1.54- \\
1.74)\end{array}$ & $\begin{array}{l}1.65(1.53- \\
1.76)\end{array}$ & $\begin{array}{l}1.52(1.41- \\
1.63)\end{array}$ & 0.015 \\
\hline \multicolumn{6}{|l|}{ FAI } \\
\hline Model 1 & $\begin{array}{l}4.16(3.54- \\
4.78)\end{array}$ & $\begin{array}{l}4.43(3.85- \\
5.02)\end{array}$ & $\begin{array}{l}4.85(4.18- \\
5.51)\end{array}$ & $\begin{array}{l}4.33(3.71- \\
4.96)\end{array}$ & 0.551 \\
\hline Model 2 & $\begin{array}{l}4.10(3.49- \\
4.71)\end{array}$ & $\begin{array}{l}4.50(3.93- \\
5.07)\end{array}$ & $\begin{array}{l}4.87(4.23- \\
5.52)\end{array}$ & $\begin{array}{l}4.30(3.69- \\
4.90)\end{array}$ & 0.517 \\
\hline Model 3 & $\begin{array}{l}4.09(3.46- \\
4.71)\end{array}$ & $\begin{array}{l}4.52(3.95- \\
5.09)\end{array}$ & $\begin{array}{l}4.89(4.24- \\
5.53)\end{array}$ & $\begin{array}{l}4.31(3.70- \\
4.93)\end{array}$ & 0.533 \\
\hline
\end{tabular}

Cl: confidence interval; TT: total testosterone; FAl: free testosterone index.

Model 1 was adjusted by age.

Model 2 was additionally adjusted by body mass index, waist circumference, systolic blood pressure and diastolic blood pressure.

Model 3 was additionally adjusted by follicle stimulating hormone, estradiol, fasting plasma glucose and fasting insulin.

Test for trend was based on variable containing median value for each quartile.

Logistic regression analysis showed that serum phosphorus levels were significantly and inversely associated with IR status in all models (Table 4). The OR (95\% Cl) for IR comparing the lowest vs. highest quartiles of serum phosphorus levels was $1.83(1.03-3.27)(P$ trend $=0.026)$ according to the multivariable model adjusted for age (model 1 ). After further adjusting for BMI, WC, SBP, and DBP (model $2)$, the corresponding OR $(95 \% \mathrm{Cl})$ was $2.27(1.20-4.29)(P$ trend $=0.007)$. And after additionally 
adjusting for FSH, LH, E2, TT and SHBG (model 3), the corresponding OR (95\% Cl) was 2.26 (1.17-4.35, $P$ trend $=0.009$ ).

Table 4

Adjusted OR $(95 \% \mathrm{Cl})$ for IR status across quartiles of serum phosphorus

\begin{tabular}{|c|c|c|c|c|c|}
\hline \multirow[t]{2}{*}{ Variables } & \multicolumn{4}{|c|}{ Quartiles of Serum Phosphorus(mmol/L) } & \multirow[t]{2}{*}{$P_{\text {trend }}$} \\
\hline & Q1 $(<0.98)$ & Q2 (0.98 - 1.12) & Q3 (1.13 - 1.24) & Q4 (>1.24) & \\
\hline Model 1 & $1.83(1.03-3.27)$ & $0.93(0.51-1.69)$ & $0.77(0.39-1.50)$ & 1 (Reference) & 0.026 \\
\hline$P$ value & 0.040 & 0.803 & 0.437 & & \\
\hline Model 2 & $2.27(1.20-4.29)$ & $1.15(0.60-2.19)$ & $0.77(0.37-1.57)$ & 1 (Reference) & 0.007 \\
\hline$P$ value & 0.012 & 0.674 & 0.465 & & \\
\hline Model 3 & $2.26(1.17-4.35)$ & $1.11(0.58-2.12)$ & $0.76(0.37-1.57)$ & 1 (Reference) & 0.009 \\
\hline$P$ value & 0.015 & 0.765 & 0.457 & & \\
\hline \multicolumn{6}{|c|}{ OR: odds ratio; Cl: confidence interval; IR: insulin resistance. } \\
\hline \multicolumn{6}{|c|}{ Model 1 was adjusted by age. } \\
\hline \multicolumn{6}{|c|}{$\begin{array}{l}\text { Model } 2 \text { was additionally adjusted by body mass index, waist circumference, systolic blood pressure } \\
\text { and diastolic blood pressure. }\end{array}$} \\
\hline \multicolumn{6}{|c|}{$\begin{array}{l}\text { Model } 3 \text { was additionally adjusted by follicle stimulating hormone, luteinizing hormone, estradiol, } \\
\text { total testosterone and sex hormone binding globulin. }\end{array}$} \\
\hline
\end{tabular}

Serum phosphorus levels were also significantly and negatively correlated with HA status in all models (Table 5). The OR $(95 \% \mathrm{Cl})$ for HA comparing the lowest vs. highest quartiles of serum phosphorus levels was $2.45(1.45-4.12)$ ( $P$ trend $=0.001)$ according to the multivariable model adjusted for age (model 1$)$. After further adjusting for BMI, WC, SBP, and DBP (model 2), the corresponding OR $(95 \% \mathrm{Cl}$ ) was 2.45 (1.44-4.42) ( $P$ trend $=0.002)$. And after additionally adjusting for FSH, E2, FPG, and FIN (model 3 ), the corresponding OR $(95 \% \mathrm{Cl})$ was $2.58(1.48-4.51)(P$ trend $=0.002)$. 
Table 5

Adjusted OR $(95 \% \mathrm{Cl})$ for HA status across quartiles of serum phosphorus

\begin{tabular}{|c|c|c|c|c|c|}
\hline \multirow[t]{2}{*}{ Variables } & \multicolumn{4}{|c|}{ Quartiles of Serum Phosphorus (mmol/L) } & \multirow[t]{2}{*}{$P_{\text {trend }}$} \\
\hline & Q1 $(<0.98)$ & Q2 (0.98 - 1.12) & Q3 $(1.13-1.24)$ & $\mathrm{Q} 4(>1.24)$ & \\
\hline Model 1 & $2.45(1.45-4.12)$ & $1.65(0.99-2.74)$ & $1.75(1.02-2.99)$ & 1 (Reference) & 0.001 \\
\hline$P$ value & 0.001 & 0.053 & 0.042 & & \\
\hline Model 2 & $2.45(1.44-4.42)$ & $1.65(0.99-2.76)$ & $1.81(1.05-3.12)$ & 1 (Reference) & 0.002 \\
\hline$P$ value & 0.001 & 0.055 & 0.032 & & \\
\hline Model 3 & $2.58(1.48-4.51)$ & $1.57(0.93-2.66)$ & $1.84(1.06-3.20)$ & 1 (Reference) & 0.002 \\
\hline$P$ value & 0.001 & 0.092 & 0.031 & & \\
\hline \multicolumn{6}{|c|}{ OR: odds ratio; Cl: confidence interval; HA: hyperandrogenemia. } \\
\hline \multicolumn{6}{|c|}{ Model 1 was adjusted by age. } \\
\hline \multicolumn{6}{|c|}{$\begin{array}{l}\text { Model } 2 \text { was additionally adjusted by body mass index, waist circumference, systolic blood pressure } \\
\text { and diastolic blood pressure. }\end{array}$} \\
\hline \multicolumn{6}{|c|}{$\begin{array}{l}\text { Model } 3 \text { was additionally adjusted by follicle stimulating hormone, estradiol, fasting plasma glucose } \\
\text { and fasting insulin. }\end{array}$} \\
\hline
\end{tabular}

\section{Discussion}

Our results showed that serum phosphorus concentrations were significantly and negatively correlated with FPG, FIN, HOMA-IR, and TT. Lower serum phosphorus concentrations were associated with higher risk of IR and worsened HA in normal-weight women with PCOS.

IR is considered to be the primary pathological basis for reproductive dysfunction in PCOS. Our result showed a negative correlation between serum phosphorus concentrations and FPG, FIN, and HOMA-IR independent of age, BMI, WC, SBP, DBP, FSH, LH, E2, TT and SHBG, and lower serum phosphorus concentrations were associated with higher risk of IR, which was similar to the results of previous studies conducted in general populations [12]. In a Japanese study of 1657 individuals, serum phosphorus concentration was inversely associated with FPG, FIN, HOMA-IR, and HOMA- $\beta$. In another larger healthy population-based study of routine checkups involving 16,041 Japanese individuals, serum phosphorus concentrations were significantly and inversely associated with FPG [14]. Furthermore, serum phosphorus concentration might also be negatively associated with FPG in the elderly in Taiwan[15]. In PCOS patients, the data in the study carried out by Mahmoudi et al. found an age, BMI, calcium, PTH, and 25(OH)D-independent and negative correlation between serum phosphorus concentrations and insulin and HOMA-IR in women with PCOS [19]. Their results are in agreement with ours; however, the subjects in 
their study were much more overweight, with an average BMI of $26.32 \mathrm{~kg} / \mathrm{m}^{2}$, whereas our patients were all normal weight $\left(\mathrm{BMI}<24 \mathrm{~kg} / \mathrm{m}^{2}\right)$. Although obesity is a common feature in PCOS patients and contributes to the association IR and PCOS, it is estimated that approximately $60 \%$ of normal-weight women with PCOS show impaired insulin sensitivity[24]. The molecular mechanisms behind the postbinding defects in the insulin signaling transduction pathway in PCOS patients have been widely investigated. A recent study suggests that PCOS presents a unique type of PCOS-related IR (intrinsic defects) due to changes in insulin action of heterogeneous factors, which can occur even in the absence of obesity. These etiological factors include polygenic inheritance and endocrine, metabolic, and immune factors that interact with environmental factors such as diet nutrient[25]. To the best of our knowledge, ours is the first study to demonstrate an inverse association between serum phosphorus levels and IR in normal-weight women with PCOS, suggesting that low phosphorus levels are likely related to PCOSassociated IR by means of obesity-independent mechanisms.

There are several ways in which phosphorus may interfere with glucose metabolism. First, phosphorus plays a crucial role in the process of energy transfer via the formation of adenosine triphosphate (ATP) [26]. Low blood phosphorus concentrations can result in decreased ATP synthesis[27], thus inducing abnormal energy metabolism, and therefore insulin-stimulated glucose utilization, which is dependent on sufficient energy sources, is reduced, ultimately promoting IR[28][29]. Second, IR is frequently associated with chronic inflammatory states and autoimmune disorders in women with PCOS. Animal experiments have demonstrated that phosphorus deficiency can induce inflammation and can impair immune function[30][31]. Thus, lower phosphorus might affect IR by mediating immune responses and inflammatory processes. Further studies are required to elucidate the role of phosphorus in glucose metabolism and insulin action among PCOS patients.

HA is another significant characteristic of PCOS patients. Our results showed that serum phosphorus concentrations were negatively with TT levels, and lower serum phosphorus concentrations were associated with worsened HA. These associations remained significant after further adjustment for FPG and FIN in the multivariate model because IR and HA intimately interact with each other, indicating that a low phosphorus level might be an independent determinant for HA in PCOS patients. The inverse association between serum phosphorus and HA is a novel and intriguing discovery in our study. These results are consistent with a previous study conducted in the US that reported that higher serum T levels are associated with lower serum phosphorus levels in older men even after adjustment for age, race, and E2 levels[16]. Wulaningsih et al. observed a similar but weaker pattern between TT and inorganic phosphate in men aged 20 years or more [32]. However, a recent cross-sectional study from Korea did not find a significant correlation between serum phosphorus and $\mathrm{T}$ in middle-aged men with erectile dysfunction[33]. These differences in results might be accounted for by different ages, races, genders, or diseases. However, little has been reported regarding the link between sex steroid hormones and phosphorus in observational studies of PCOS patients, thus our finding is needed to be confirmed in future studies. 
The inverse association between serum phosphorus and TT levels in our study might have different explanations. First, it might be a secondary result of serum phosphorus and IR because IR and HA are interactional in PCOS. In addition, it is known that T is the primary precursor of E2 synthesis, and serum estrogen affects the balance of bone metabolism, which demands plenty of phosphorus[34]. However, we suspect that the association between phosphorus and TT might not be directly mediated by E2 because this association remains significant after adjusting for FSH and E2 in the multivariable model analysis. On the other hand, we assumed that gastrointestinal absorption, bone metabolism, and renal excretion might influence the association between serum phosphorus and TT levels. Due to the complexity of PCOS and because the role of serum phosphorus in the regulation of T production has not been widely investigated, further research is needed to elucidate the mechanism behind the association between serum phosphorus and TT.

Based on the findings described above, it is reasonable to speculate that phosphorus may have a promising effect on PCOS. Unfortunately, no study is available on phosphorus supplementation for PCOS patients, and only a few studies have been performed in non-PCOS populations. Previous results from an international study showed that dietary phosphorus is inversely associated with blood pressure and suggested the potential effect of increased phosphorus intake on lowering blood pressure [35]. Recently, the results of a randomized controlled trial (RCT) conducted in overweight/obese adults indicated that phosphorus supplementation for 12 weeks could significantly decrease weight, BMI, WC, and subjective appetite scores[36]. Another RCT demonstrated that 6 months of T therapy could reduce serum phosphate levels in hypogonadal men [37]. In this context, we hypothesize that raising blood phosphorus via phosphorus supplementation could reverse the HA seen in PCOS. In addition, sufficient dietary phosphorus intake has been shown to have a favorable effect on the immune system and inflammation in some animal studies[38][39]. In summary, these findings support a promising role for phosphorus in the management of hypertension, obesity, HA, and inflammation, which are also prevalent in PCOS patients. In the future, larger RCTs are recommended to be conducted to determine the potential therapeutic effect in PCOS patients.

Our study has several strengths. This was the first study to investigate the effect of phosphorus on IR and HA in PCOS patients, and our study included a large sample size that was highly homogeneous and was a multicenter-based population. In addition, some potential confounding factors were fully adjusted for. Furthermore, all samples for phosphorus measurements were taken on the morning after an overnight of fasting in order to reduce the influences of diurnal variation.

There were also some limitations. First, this was a cross-sectional study, and it was not clear whether low phosphorus concentration was a consequence of IR and HA or a contributor. Second, nutritional records such as dietary phosphorus intake were not measured in our study. Third, we did not use the goldstandard glucose clamp method to evaluate IR. Fourth, our cohort consisted entirely of Chinese patients, and caution should be taken in extrapolating these results to other ethnic groups.

\section{Conclusions}


In conclusion, our data demonstrated that serum phosphorus concentrations were significantly and negatively correlated with FPG, FIN, HOMA-IR, and TT. Lower serum phosphorus levels might play a contributing role in the development of IR and HA in normal-weight PCOS patients. Our study thus provides new insights into the pathogenesis of PCOS. However, further prospective studies are required to confirm this finding and the underlying mechanisms. Future RCTs should also be conducted to assess whether phosphorus supplementation or dietary phosphorus intake have a beneficial effect in PCOS patients.

\section{Declarations}

\section{DATA AVAILABILITY STATEMENT}

All of the original data were described in the main text and Supplemental Materials. Any other questions can be directed to the corresponding author: Xiaoke Wu,2417956638@qq.com.

\section{AUTHOR CONTRIBUTIONS}

Conceptualization, S-KZ and HH; Methodology, JC; Software, JL; Validation, S-KZ, HH, and X-KW; Formal Analysis, HH; Investigation, S-KZ; Resources, X-KW; Data Curation, YW, JC, and J-SG; Writing-Original Draft Preparation, S-KZ and HH; Writing-Review \& Editing, JL and X-KW; Visualization, J-XF and M-YZ; Supervision, X-KW; Project Administration, YW, JC and J-SG; Funding Acquisition, X-KW, YW, JC.

\section{CONFLICTS OF INTEREST}

The authors declare no conflict of interest.

\section{FUNDING}

This study was supported by (1) the National Key R\&D Program of China, Research and development for modernization of Chinese medicine, Evidence-based evaluation of the program of integration of traditional Chinese and western medicine for the high incidence of gynecological diseases (No.2019YFC1709500); (2) the National clinical cooperation pilot program of TCM and western medicine for major and difficult diseases (National office of traditional Chinese medicine, No.[2018]3), Combined traditional Chinese and western medicine in infertility and assisted reproductive technology; (3) the Scientific Research Fund of Heilongjiang University of Chinese Medicine, Study on Acupuncture Combined with Traditional Chinese Medicine Compound to Improve the Live Birth Rate of Patients with in Vitro Fertilization and Embryo Transfer (No. 2019BS09); (4) Natural Science Foundation of Heilongjiang Province of China (LH2019H046). 


\section{ACKNOWLEDGMENTS}

The Steering Committee members of PCOSAct included Xiao-Ke Wu, Jiang-Ping Liu, Tai-Xiang Wu, Ernest HY Ng, Elisabet Stener-Victorin, Heping Zhang, and Richard S Legro (Chair). The Data and Safety Monitoring Board members included Esther Eisenberg, Wei-Liang Weng, Su-Lun Sun, Wei Zou, Zi-Dan Chen, and Robert Rebar (Chair). Meizhuo Zhang of Yale contributed to the randomization scheme and training of our study personnel. Jin-Ying Fu, Chang-Ling Zhu, and Xiao-Hong Wang participated in the patient recruitment at local sites in Henan, Wenzhou, and Xuzhou. Other personnel with administrative resource support included Song-Jiang Liu, Gui-Yuan Wang, Yan-Qiu Du, Yang Xia, Shu-Lai Li, Ke-Qiu Zhang, and Jian-Hua Shen. Yan Li, Wen-Juan Shen, Wei Li, and Jing Cong were involved in protocol preparation and blood sample management in the Harbin office and in the core laboratory. We thank the Reproductive Medicine Network Steering Committee of the National Institutes of Health for sharing the protocol and case-report forms from the Pregnancy in Polycystic Ovary Syndrome II study.

\section{References}

1. Woodward A, Klonizakis M, Broom D. Exercise and Polycystic Ovary Syndrome. Adv Exp Med Biol. 2020; 1228:123-136.

2. Anagnostis $P$, Tarlatzis BC, Kauffman RP. Polycystic ovarian syndrome (PCOS): Long-term metabolic consequences. Metabolism. 2018; 86:33-43.

3. Helvaci N, Yildiz BO. Polycystic ovary syndrome and aging: Health implications after menopause. Maturitas. 2020; 139:12-19.

4. Bannigida DM, Nayak BS, Vijayaraghavan R. Insulin resistance and oxidative marker in women with PCOS. Arch Physiol Biochem. 2020;126(2):183-186.

5. Stepto NK, Hiam D, Gibson-Helm M, et al. Exercise and insulin resistance in PCOS: muscle insulin signalling and fibrosis. Endocr Connect. 2020; 9(4):346-359.

6. Glueck CJ, Goldenberg N. Characteristics of obesity in polycystic ovary syndrome: Etiology, treatment, and genetics. Metabolism. 2019; 92:108-120.

7. Wang J, Wu D, Guo H, et al. Hyperandrogenemia and insulin resistance: The chief culprit of polycystic ovary syndrome. Life Sci. 2019; 236: 116940.

8. Yin J, Hong X, Ma J, et al. Serum Trace Elements in Patients with Polycystic Ovary Syndrome: A Systematic Review and Meta-Analysis. Front Endocrinol (Lausanne). 2020; 11: 572384.

9. Peacock M. Phosphate Metabolism in Health and Disease. Calcif Tissue Int. 2021;108(1):3-15.

10. Khalil R, Kim NR, Jardi F, et al. Sex steroids and the kidney: role in renal calcium and phosphate handling. Mol Cell Endocrinol. 2018; 465:61-72.

11. Billington EO, Gamble GD, Bristow $\mathrm{S}$, et al. Serum phosphate is related to adiposity in healthy adults. Eur J Clin Invest. 2017;47(7):486-493. 
12. Akter S, Eguchi M, Kochi T, et al. Association of Serum Calcium and Phosphate Concentrations with Glucose Metabolism Markers: The Furukawa Nutrition and Health Study. Nutrients. 2020;12(8):2344.

13. Lorenzo C, Hanley AJ, Rewers MJ, et al. Calcium and phosphate concentrations and future development of type 2 diabetes: the Insulin Resistance Atherosclerosis Study. Diabetologia. 2014;57(7):1366-1374.

14. Shimodaira M, Okaniwa S, Nakayama T. Reduced Serum Phosphorus Levels Were Associated with Metabolic Syndrome in Men but Not in Women: A Cross-Sectional Study among the Japanese Population. Ann Nutr Metab. 2017;71(3-4):150-156.

15. Jhuang YH, Kao TW, Peng TC, et al. Serum Phosphorus as a Risk Factor of Metabolic Syndrome in the Elderly in Taiwan: A Large-Population Cohort Study. Nutrients. 2019;11(10):2340.

16. Meng J, Ohlsson C, Laughlin GA, et al. Associations of estradiol and testosterone with serum phosphorus in older men: the Osteoporotic Fractures in Men study. Kidney Int. 2010;78(4):415-422.

17. Lopez DS, Wulaningsih W, Tsilidis KK, et al. Environment-wide association study to comprehensively test and validate associations between nutrition and lifestyle factors and testosterone deficiency: NHANES 1988-1994 and 1999-2004. Hormones (Athens). 2020;19(2):205-214.

18. Ellam T, Wilkie $M$, Chamberlain $\mathrm{J}$, et al. Dietary phosphate modulates atherogenesis and insulin resistance in apolipoprotein E knockout mice--brief report. Arterioscler Thromb Vasc Biol. 2011;31(9):1988-1990.

19. Mahmoudi T, Gourabi H, Ashrafi M, et al. Calciotropic hormones, insulin resistance, and the polycystic ovary syndrome. Fertil Steril. 2010;93(4):1208-1214.

20. Kuang H, Li Y, Wu X, et al. Acupuncture and clomiphene citrate for live birth in polycystic ovary syndrome: study design of a randomized controlled trial. Evid Based Complement Alternat Med. 2013:527303.

21. Wu XK, Stener-Victorin E, Kuang HY, et al. Effect of Acupuncture and Clomiphene in Chinese Women with Polycystic Ovary Syndrome: A Randomized Clinical Trial. JAMA. 2017;317(24):2502-2514.

22. Zhou B; Coorperative Meta-Analysis Group of China Obesity Task Force. [Predictive values of body mass index and waist circumference to risk factors of related diseases in Chinese adult population]. Zhonghua Liu Xing Bing Xue Za Zhi. 2002;23(1):5-10. Chinese.

23. Xing XY, Yang WY, Yang ZJ. The Diagnostic Significance of Homeostasis Model Assessment of Insulin Resistance in Metabolic Syndrome Among Subjects with Different Glucose Tolerance. Chin J Diabetes. 2004; 12(3):182-186.

24. Moghetti P, Tosi F. Insulin resistance and PCOS: chicken or egg? J Endocrinol Invest. 2021;44(2):233244.

25. Diamanti-Kandarakis E, Dunaif A. Insulin resistance and the polycystic ovary syndrome revisited: an update on mechanisms and implications. Endocr Rev. 2012 Dec;33(6):981-1030.

26. Müller WEG, Wang S, Neufurth $M$, et al. Polyphosphate as a donor of high-energy phosphate for the synthesis of ADP and ATP. J Cell Sci. 2017;130(16):2747-2756. 
27. Pesta DH, Tsirigotis DN, Befroy DE, et al. Hypophosphatemia promotes lower rates of muscle ATP synthesis. FASEB J. 2016;30(10):3378-3387.

28. Bódis K, Roden M. Energy metabolism of white adipose tissue and insulin resistance in humans. Eur J Clin Invest. 2018;48(11): e13017.

29. Kupriyanova $Y$, Zaharia OP, Bobrov $P$, et al. Early changes in hepatic energy metabolism and lipid content in recent-onset type 1 and 2 diabetes mellitus. J Hepatol. 2021;74(5):1028-1037.

30. Chen K, Jiang WD, Wu P, et al. Effect of dietary phosphorus deficiency on the growth, immune function and structural integrity of head kidney, spleen and skin in young grass carp (Ctenopharyngodon idella). Fish Shellfish Immunol. 2017; 63:103-126.

31. Chen $K$, Zhou XQ, Jiang WD, et al. Impaired intestinal immune barrier and physical barrier function by phosphorus deficiency: Regulation of TOR, NF-KB, MLCK, JNK and Nrf2 signalling in grass carp (Ctenopharyngodon idella) after infection with Aeromonas hydrophila. Fish Shellfish Immunol. 2018; 74:175-189.

32. Wulaningsih W, Van Hemelrijck M, Michaelsson K, et al. Association of serum inorganic phosphate with sex steroid hormones and vitamin $\mathrm{D}$ in a nationally representative sample of men. Andrology. 2014; 2(6):967-76.

33. Min SK, Choi K, Kim SK, et al. Phosphorus as predictive factor for erectile dysfunction in middle aged men: A cross sectional study in Korea. Investig Clin Urol. 2016;57(6):442-448.

34. Xu D, Teng X, Guo R, et al. Metabonomic analysis of hypophosphatemic laying fatigue syndrome in laying hens. Theriogenology. 2020; 156:222-235.

35. Elliott $P$, Kesteloot $H$, Appel LJ, et al. Dietary phosphorus and blood pressure: international study of macro- and micro-nutrients and blood pressure. Hypertension. 2008;51(3):669-675.

36. Ayoub JJ, Samra MJ, Hlais SA, et al. Effect of phosphorus supplementation on weight gain and waist circumference of overweight/obese adults: a randomized clinical trial. Nutr Diabetes. 2015;5(12): e189.

37. Pedersen L, Christensen LL, Pedersen SM, et al. Reduction of calprotectin and phosphate during testosterone therapy in aging men: a randomized controlled trial. J Endocrinol Invest. 2017;40(5):529-538.

38. Q, Xu M, Sun Z, et al. Effects of dietary monocalcium phosphate supplementation on the antioxidative capacity, anti-bacteria function in immune organs of obscure puffer (Takifugu obscurus) after infection with Aeromonas hydrophila. Fish Shellfish Immunol. 2020; 98: 843-852.

39. Kokou F, Sarropoulou E, Cotou E, et al. Effects of graded dietary levels of soy protein concentrate supplemented with methionine and phosphate on the immune and antioxidant responses of gilthead sea bream (Sparus aurata L.). Fish Shellfish Immunol. 2017; 64: 111-121.

\section{Supplementary Files}

This is a list of supplementary files associated with this preprint. Click to download. 
- Supplementarymaterial.docx

Page 19/19 